\title{
FORUM EDITORIAL
}

\section{Novel Faces of Heme Oxygenase-1: Mechanisms and Therapeutic Potentials}

\author{
Jozef Dulak and Alicja Jozkowicz
}

\begin{abstract}
Not only double, Janus face, but numerous appearances characterize heme oxygenase-1 (HO-1), an inducible enzyme which main role is to degrade heme. Recently, the noncanonical functions of HO-1 have particularly attracted researchers' attention. Indeed, understanding the enzyme-independent activities of HO-1 can provide additional chances for translational application of research on HO-1. In this Forum, eight reviews and two original articles describe a plethora of mechanisms in which this pleiotropic protein is involved. Further understanding of HO-1 functions is of particular significance for elucidating the pathology of various human diseases and providing rationale for novel therapies. Antioxid. Redox Signal. 20, 1673-1676.
\end{abstract}

$\mathbf{S}^{\mathrm{s}}$ INCE THE DISCOVERY of heme oxygenase in 1967, the understanding of this very important enzymatic pathway in mammals has increased enormously. Majority of research concerns the inducible form, heme oxygenase-1 (HO-1), encoded by the Hmox-1 gene, and covers areas related to probably all physiological and pathological conditions. The animal models of Hmox-1 knockout reflect, although not completely, the conditions found in two described, so far, cases of humans devoid of functional Hmox-1 alleles. The effect of HO-1 deficiency is significant, both in humans and mice, and this additionally proves the importance of this enzyme. However, data in recent years accumulate, indicating that the functions of HO-1 extend beyond the effect directly related to heme degradation. The noncanonical roles of HO- 1 have been addressed in numerous reviews published on that subject. However, the time is ready for new synthesis and the present Forum aims to discuss several aspects of HO-1, which have not been addressed in other articles.

This Forum consists of eight reviews and two original articles. Both classical functions of HO-1 are addressed, as well as the new ones, particularly related to the role of HO-1 in stem cell differentiation and the novel molecular mechanisms, involving the HO-1 effect on microRNAs.

The classical function of HO- 1 is to degrade heme. This aspect is discussed and elaborated from different perspec- tives in the review written by Gozzelino and Soares (4). The authors concentrate on iron, one of the three products of HO-1 activity. Iron is a very reactive molecule, therefore its binding to the porphyrin ring in the form of iron protoporphyrin-heme, is the first way to prevent iron cytotoxicity. More than $80 \%$ of bioavailable iron in the cell is bound in this way. However, this solution is not permanent, heme proteins are degraded, and heme is released. Iron in Fenton reaction can generate noxious hydroxyl radicals. Not surprisingly, the next series of mechanisms has evolved, helping to eliminate iron from the cells or binding it to another protein-ferritin. The review by Gozzelino and Soares (4) elaborates particularly on one of the special functions of ferritin, which is the regulation of immune responses.

As mentioned, the importance of HO-1 goes beyond its enzymatic function, and HO-1 can be cytoprotective even if there might not be sufficient access to its substrate heme. Indeed, in such a case, as Phyllis Dennery pointed out in her review published in this Forum, "is unlikely that the $\mathrm{HO}$ reaction can mediate cytoprotective effects via its byproducts." Therefore, one can expect that HO-1, like numerous proteins, exerts many other effects that are not related to its classical function. Interestingly, the HO-1 protein devoid of histidine 25 , the crucial amino acid in heme-binding pocket, has been demonstrated to protect against hydrogen

Department of Medical Biotechnology, Faculty of Biochemistry, Biophysics and Biotechnology, Jagiellonian University, Krakow, Poland. 
peroxide-induced cytotoxicity (see references in review by Dennery, this Forum). Further research, coming mostly from Dennery's laboratory, and supported by others, demonstrated unexpected localization of $\mathrm{HO}-1$ protein in the nucleus, where it presumably cannot perform its enzymatic activity due to the lack of cytochrome $\mathrm{P} 450$ reductase (CPR), a necessary donor of electrons for HO-1. Further studies have shown that the nuclear form is shorter than native $32 \mathrm{kDa}$ HO-1, being devoid of C-end, and may bind certain transcription factors to regulate the expression of several genes (see references in reviews by Dennery and by Dunn et al., this Forum).

The new studies, coming from the Roland Stocker laboratory and discussed extensively in review by Dunn et al. (2), underlie also that the nuclear form of HO-1 can play really important functions. The authors discuss a plethora of potential mechanisms of HO-1 biological roles not related to heme degradation, addressing particularly the significance of various intracellular locations of HO-1. Further research should elucidate whether nucleuslocalized HO-1 is enzymatically active or not. As mentioned, the nuclear form may be truncated, however, this does not preclude its enzymatic activity. A matter of debate are the concerns of the already mentioned lack of $\mathrm{CPR}$ in the nucleus, as well as the presence of heme in the nucleus. The suggestions that biliverdin reductase could transport heme to the nucleus and that ascorbate can replace $\mathrm{CPR}$ as the electron donor are the interesting possibilities remaining to be confirmed [for references see Dunn et al. (2)].

Both enzymatic activity of $\mathrm{HO}-1$, the products released, as well as the noncanonical functions of HO-1 can regulate other processes discussed in the next review of this Forum. Hull et al. extensively elaborate on the involve- ment of HO-1 in the regulation of the functions of the mononuclear phagocyte system (6). The significance of TLR-dependent signaling is addressed, as well as the role of HO-1 played in different subpopulations of macrophages. The authors deliberate also on the controversial issue such as whether inhibition of dendritic cell maturation is dependent on the HO-1 activity. Further studies should elucidate also whether exogenous $\mathrm{CO}$ reproduces the activity of endogenous, heme-derived $\mathrm{CO}$, generated by $\mathrm{HO}-1$ and $\mathrm{HO}-2$, or whether the effect of delivered $\mathrm{CO}$ is rather an unrelated phenomenon, however, worth investigating for therapeutic purposes.

Nevertheless, application of substances able to release CO is strongly considered and the review by Motterlini and Foresti (8) addresses particularly this Forum. The authors, known as the creators of carbon monoxide releasing molecules (CORMs), discuss numerous results already available on both chemistry and biology of CORMs. They, however, elaborate also on HO-1 as a potential target for new drugs, presenting the intriguing data on the mechanisms of actions of numerous activators of Hmox- 1 expression. Among them there is still a large interest in search for so-called "natural" compounds, which may induce HO-1 expression via different mechanisms.

The area in which the role of HO-1 was particularly investigated involves its effect on both the functions of cardiovascular system as well as the formation of new blood vessels. The first aspects are widely discussed by Calay and Mason (1), who present the overview on the mechanisms of induction of Hmox-1 expression and the cytoprotective and angiogenic roles of HO-1 and its products in blood vessels. Of note, the significance of those studies extends not only to cardiovascular diseases, but concerns also the role of HO-1 in cancer and other

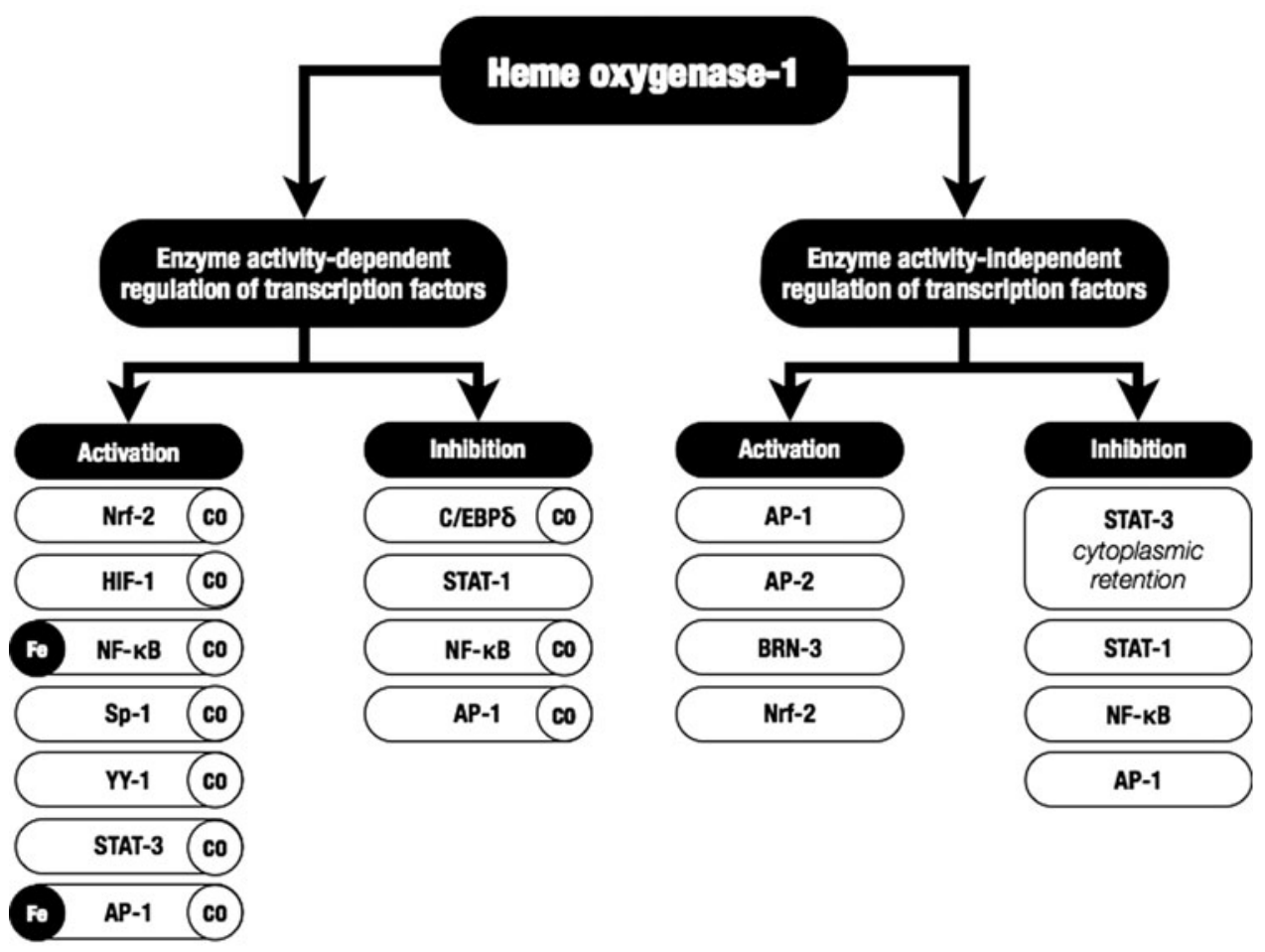

FIG. 1. Regulation of transcription factor activity by heme oxygenase-1 (HO1) and its products. The enzyme activity-independent action of HO-1 can be mediated both by its full and truncated form. In different experimental conditions, either activation or inhibition of the same transcription factors (like AP-1, NF- $\kappa \mathrm{B}$, or STAT-3) has been observed (based on data in the articles cited in this Forum). 
morbidities. This will be undoubtedly the matter of future debates, and some new directions are indicated in the review by Wegiel et al. (9), which raises the potential involvement of HO-1 in the control of metabolism. It can be hypothesized that better understanding of the role of HO- 1 in regulation of metabolism, particularly in neoplastic tissues, may help to elucidate potentially discrepant data, such as the those concerning the effect of HO-1 on various tumor growth. This is not a trivial issue, as in fact, as Dunn and coworkers indicate in their review as follows: "any capacity to modulate HMOX1 in cardiovascular and metabolic diseases must be tempered with an appreciation that HMOX1 may impact on cancer."

It is probable, that the cell-specific effect of HO-1 is at least partially dependent on microRNAs, known to work often in such a manner. Recent data, particularly from our team, indicate another important mechanism of action of HO-1. It appears that HO-1 is a potent regulator of microRNA expression and processing. This is of relevance to the recent widely investigated involvement of HO-1 in stem cell differentiation [reviewed in Kozakowska et al. (7)]. Both HO-1 and Nrf2, its upstream transcription factor, are necessary also for survival, proliferation, migration, and differentiation of cells previously called endothelial progenitor cells, being, in fact, rather proangiogenic monocytes, as demonstrated in the two original articles published in this Forum $(3,5)$. We believe those works may add to several previous studies indicating the significance of HO-1 in blood vessel formation in reparative processes, which may partially depend on those cells [reviewed in this Forum: $(1,2)$ ]. However, their progenitor/stem cell nature is currently the matter of strong debate and new data indicate that those bone marrow cells have to be considered rather as proangiogenic cells (PACs), not having the special stem cell properties. Moreover, two studies published in this Forum $(3,5)$ indicate that PACs may not contribute (directly) to inflammation-driven revascularization. Interestingly, despite the impairment of their mobilization from bone marrow, as evidenced in the Nrf2-knockout mice, the regeneration of blood vessels after hindlimb ischemia was, in fact, accelerated in those mice in comparison with wild-type individuals (3). In contrast, in the second study, injection of PACs did not improve and even worsened the regeneration process in the hindlimb ischemia or wound healing. However, the cells may play a beneficial, but rather paracrine effect, as evidenced by the influence of PAC-conditioned media on restoration of blood perfusion (5).

In light of increasing evidence on differential intracellular localization of HO-1, and particularly its effect on transcription factors (Fig. 1), further studies should elucidate those aspects. If HO- 1 shuttles between the cytoplasm and the nucleus, it may affect the stability and activity of transcription factors, which behave in a similar way. Additionally, the reported localization of HO-1 in mitochondria raises further issues not only on regulation of gene expression, but also on the influence of HO- 1 on cellular respiration. The potential interactions between HO-1 and miR-378, a miRNA reported to be involved in cellular metabolisms [see references in Kozakowska et al. (7)], open new fascinating areas worth investigating in physiological and pathological conditions, such as cancer, muscular dystrophy, inflammation, or angiogenesis.

The effect of HO-1, both as the protein (either enzymatically active or not) and via its product on different transcription factors (Fig. 1) will certainly be the subject of intense studies in the near future. One can expect that during the next conference on heme oxygenases, scheduled for October 2014 in Sydney (http://hemeoxygenase2014.org.au), more data will be presented on this fascinating, never ending story of $\mathrm{HO}-1$ activity. It is also a reasonable hope that such a research can be translated to clinical applications. The search for specific inhibitors of HO-1 activity is necessary and may help to find new ways to treat cancers, in which HO-1 affects tumor growth, cell differentiation, angiogenesis, and metastasis, via different mechanisms discussed in this Forum.

\section{Acknowledgments}

The authors' research is supported by grants from the National Science Center (grant No: 2012/06/A/NZ1/00004 (MAESTRO), 2012/07/B/NZ1/02881 (OPUS), and 2012/06/ M/NZ1/00008 (HARMONIA).

\section{References}

1. Calay D and Mason JC. The multifunctional role and therapeutic potential of HO-1 in the vascular endothelium. Antioxid Redox Signal 20: 1789-1809, 2014.

2. Dunn LL, Midwinter RG, Ni J, Hamid HA, Parish CR, and Stocker R. New insights into intracellular locations and functions of heme oxygenase-1. Antioxid Redox Signal 20: 1723-1742, 2014.

3. Florczyk U, Jazwa A, Maleszewska M, Mendel M, Szade K, Kozakowska M, Grochot-Przeczek A, Viscardi M, Czauderna S, Bukowska-Strakova K, Kotlinowski J, Jozkowicz A, Loboda A, and Dulak J. Nrf2 regulates angiogenesis: effect on endothelial cells, bone marrow-derived proangiogenic cells and hind limb ischemia. Antioxid Redox Signal 20: 1693-1708, 2014.

4. Gozzelino R and Soares MP. Coupling heme and iron metabolism via ferritin H chain. Antioxid Redox Signal 20: 1754-1769, 2014.

5. Grochot-Przeczek A, Kotlinowski J, Kozakowska M, Starowicz K, Jagodzinska J, Stachurska A, Volger OL, Bukowska-Strakova K, Florczyk U, Tertil M, Jazwa A, Szade K, Stepniewski J, Loboda A, Horrevoets AJ, Dulak J, and Jozkowicz A. Heme oxygenase-1 is required for angiogenic function of bone marrow-derived progenitor cells: role in therapeutic revascularization. Antioxid Redox Signal 20: 1677-1692, 2014.

6. Hull TD, Agarwal A, and George J. The mononuclear phagocyte system in homeostasis and disease: a role for heme oxygenase-1. Antioxid Redox Signal 20: 1770-1788, 2014.

7. Kozakowska M, Szade K, Dulak J, and Jozkowicz A. Role of heme oxygenase-1 in postnatal differentiation of stem cells: a possible cross-talk with microRNAs. Antioxid Redox Signal 20: 1827-1850, 2014.

8. Motterlini R and Foresti R. Heme oxygenase-1 as a target for drug discovery. Antioxid Redox Signal 20: 1810-1826, 2014. 
9. Wegiel B, Nemeth Z, Correa-Costa M, Bulmer A, and Otterbein L. Heme oxygenase-1: a metabolic nike. Antioxid Redox Signal 20: 1709-1722, 2014.

Address correspondence to: Prof. Jozef Dulak Department of Medical Biotechnology Faculty of Biochemistry, Biophysics and Biotechnology

Jagiellonian University Gronostajowa 7 30-387 Krakow Poland

E-mail: jozef.dulak@uj.edu.pl
Prof. Alicja Jozkowicz. Department of Medical Biotechnology Faculty of Biochemistry, Biophysics and Biotechnology Jagiellonian University Gronostajowa 7 30-387 Krakow Poland E-mail: alicja.jozkowicz@uj.edu.pl

Date of first submission to ARS Central, December 15, 2013; date of acceptance, December 16, 2013.

Abbreviations Used
CORMs $=$ carbon monoxide releasing molecules
$\mathrm{CPR}=$ cytochrome $\mathrm{P} 450$ reductase
$\mathrm{HO}-1=$ heme oxygenase- 1
$\mathrm{PACs}=$ proangiogenic cells

